\title{
Field tests and computational simulations of the explosion of buried charges
}

\author{
Eve Roger ${ }^{1, a}$, Benjamin Loret $^{2}$, and Jean Paul Calvel ${ }^{1}$ \\ ${ }^{1}$ DGA Techniques terrestres, Rocade Est, Echangeur de Guerry, 18000 Bourges Cedex, France \\ ${ }^{2}$ Laboratoire 3SR, Domaine Universitaire, BP. 53, 38041 Grenoble Cedex 9, France
}

\begin{abstract}
Modelling buried explosion is a matter of concern for vehicle protection. Indeed, in the battlefield, Improvised Explosive Devices (IEDs) are one of the major threats for land vehicles and, more specifically, for their underbelly. Two series of field tests using several masses of explosives have been performed, varying certain geometrical parameters, the nature and the physical properties of the soil. These controlled tests have shown that the impulse transmitted to the vehicle is a function of the saturation of the soil as well as of depth of burial of the explosive. In an effort to simulate the phenomena that take place during the explosions, these tests have been used to feed the data requested in computational simulations in a finite element context. Soil modelling presents its own difficulties, especially because soil is a porous medium and the three phases (solid grains, water and air) must be considered. A non linear viscoplastic cap model has been developed where the degree of saturation is variable. The yield surface includes a failure part, a cap and a tension cutoff. Soil stiffening associated with the air expulsion has been observed to be an important aspect of the model.
\end{abstract}

\section{Introduction}

In the last few years, Improvised Explosive Devices (IED) became one of the major threats on theatres of operations. Some of them can be buried and impact the belly of vehicles. However the type of soil, whether it is cohesive or not, its water content and other parameters like the depth of burial of the explosive device largely influence the energy released by the detonation and thus the impulse transmitted to a vehicle and the deformations of its hull.

Several experimental researches have addressed the issue: Hlady [1] and Peles et al. [2] performed buried experiments in fine and coarse grained soils in dry and saturated conditions. The type of soil influences the energy transmitted by the detonation. Fine grained soils form chunks that contribute significantly to the impulse and kinetic energy (Hlady [1]). Sand projections provide much less energy than fine grained soil ejecta.

Moreover a soil with a higher density and/or a moisture content transfers more energy. Zakrisson et al. [4] highlighted that the water content of the soil influences not only the impulse transmitted by the detonation but also the deformation of a plate standing above the buried explosion. For a sandy soil, Assaf et al. [5] showed that when the degree of saturation is close to 1 , the difference is really significant on the impulse. Besides a shot in water provides the largest value of impulse and deformation (Fourney et al. [3]).

The depth of burial has also an influence on impulse and deformation. As the depth of burial of the explosive increases, the aerial pressures decrease. However, the kinetic energy transmitted upwards increases with the

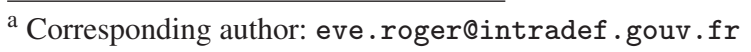

thickness of the soil above the explosive (Hlady [1], Zakrisson et al. [4]). Still, there exists a depth of burial at which the impulse is the largest. Beyond this value, increasing the depth of burial results in a decrease of impulse. This depth is directly linked to the quantity of explosive. As for the deformation, Zakrisson et al. [4] showed that it decreases as the depth of burial increases, because it is mostly associated with the shock wave and less with the impact of projections.

Experiments involving vehicles are expensive. Modelling can be a good compromise if the numerical simulations are validated by experiments. However soil modelling presents its own difficulties because of the composition of the soil which is a porous medium composed of three phases (solid particles, water and air). The three phases are important to be considered to model a buried explosion: while the air is expelled during the detonation, the water is trapped in the soil because the detonation is a so quick phenomenon that water does not have the time to escape.

A soil model has been developed taking into account the influence of the water content. Two experimental campaigns have been carried out to provide data to validate the simulations. The first campaign involved small scale detonations at three depths in dry and saturated sandy gravel. These experiments are close to the work of Bergeron et al. [6]. Pressures have been recorded and the propagation of the shock wave has been tracked by shadowgraphy. The second experimental campaign addressed the impulse transmitted by buried explosions to a test rig and the deformations of plates. Attention has been laid on the influences of the depth of burial of the explosive, of the stand-off distance and of the water content of the soil on the impulse and deformation of plates.

This is an Open Access article distributed under the terms of the Creative Commons Attribution License 4.0, which permits unrestricted use, distribution, and reproduction in any medium, provided the original work is properly cited. 
The same STANAG soil has been used in the two experimental campaigns. It is defined in the NATO procedures AEP 55 [7] by a granulometry, a density and a water content related to the Proctor optimum:

- $100 \%$ of the mass of the soil particles must pass the $40 \mathrm{~mm}$ sieve;

- between 60 and $40 \%$ must pass the $5 \mathrm{~mm}$ sieve;

- a maximum of $10 \%$ must pass the $80 \mu \mathrm{m}$ sieve;

- a density of $2200 \pm 100 \mathrm{~kg} \cdot \mathrm{m}^{-3}$ and a water content less than $1.5 \%$ of the optimum Proctor are required.

While the requirements on the granulometry were respected during the experiments, various densities and water contents were tested.

\section{A non linear elastic viscoplastic cap model}

A viscoplastic cap model with an isotropic hardening has been developed from the existing model of Tong and Tuan [8]. It is associated with a stiffening curve depending on water content to model non linear elasticity. The initial state of the soil is taken into account with the initial void ratio and the bulk modulus at zero stress. The model was written in a user-defined material subroutine and incorporated in LS Dyna [9].

\subsection{Overview of the model}

The constitutive yield surface is an extension of the Drucker-Prager model. Time dependence (viscoplasticity) is obtained by Perzyna's overstress idea.

The yield surface is divided into three regions, Fig. 1: a cap surface $f_{1}$ (Eq. (1)) for $I_{1}>\mathrm{L}$ which can harden when stresses increase and has the form of an ellipse, a failure surface $f_{2}$ (Eq. (2)) for $0<I_{1}<\mathrm{L}$ which limits shear, and a cutoff surface $f_{3}$ (Eq. (3)) for $I_{1}<0$ of elliptical shape to control tensile stresses. Attention has been laid to ensure continuity of the outward normals at the transition between two surfaces.

$$
\begin{gathered}
f_{1}\left(I_{1}, \sqrt{J_{2}}\right)=\sqrt{J_{2}+\frac{1}{R^{2}}\left(I_{1}-M\right)^{2}}-\frac{1}{R}(X-M) \\
f_{2}\left(I_{1}, \sqrt{J_{2}}\right)=\sqrt{J_{2}}+\alpha-\gamma \exp \left(-\beta I_{1}\right)+\theta I_{1} \\
f_{3}\left(I_{1}, \sqrt{J_{2}}\right)=\sqrt{J_{2}+\frac{B^{2}}{A^{2}}\left(I_{1}-(A-T)\right)^{2}}-B \\
A=T \frac{T d f_{2} / d I_{1}(0)-f_{2}(0)}{2 T d f_{2} / d I_{1}(0)-f_{2}(0)} \\
B=T \frac{A^{2}}{A-T} \sqrt{1-\frac{(A-T)^{2}}{A^{2}}} \frac{d f_{2}}{d I_{1}}(0) .
\end{gathered}
$$

In the above equations, $I_{1}$ is the first stress invariant, $J_{2}$ is the second deviatoric stress invariant, $X$ corresponds to the intersection between the cap surface and the $I_{1}$ axis, $M$ is the point of the cap with a horizontal tangent, $L$ is the value of $I_{1}$ such as $f_{1}(L)=f_{2}(L)$ and $\alpha, \beta, \gamma$ and $\theta$ are soil parameters. $T$ is the limit tensile stress.
The hardening law for the cap surface uses the viscoplastic volume change as a hardening variable:

$$
X(k)-X(0)=\frac{1}{D}\left(\left|1-\frac{k}{W}\right|^{-1}-1\right), \quad k=-\operatorname{tr} \boldsymbol{\varepsilon}_{v p} .
$$

The parameter $W$ is the volumetric fraction of void in the soil and $D$ a material parameter. Altogether, the model requires 12 parameters.

\subsection{Stiffening during air expulsion}

The detonation of explosives involves very large stresses in the soil of the order of the gigapascal. Elastic moduli highly depend on stresses: De [10] and Zakrisson et al. [4] showed that elastic moduli could be multiplied by more than 1000 when the soil undergoes such large stresses. A constitutive stiffening of the elastic properties was therefore developed.

During a detonation, while the air is expelled, the water is trapped in the soil because the detonation is so fast that it does not have the time to escape. Therefore, as the stresses increase in the soil, the three phase medium progressively turns to a two phase medium composed of soil particles and water. In the fully compacted state, the elastic properties of the soil-water mixture are obtained by averaging the elastic properties of soil and water by their volume fractions.

The bulk modulus of the crushed soil matter $K_{w g}$ therefore depends on the bulk modulus of water $K_{w}$ (around $2 \mathrm{GPa}$ ) and solid particles $K_{g}$ (around $36 \mathrm{GPa}$ ):

$$
K_{w g}(w)=K_{g}\left(1-\sqrt{n_{w}^{\infty}}\right)^{2}+K_{w} n_{w}^{\infty}
$$

with $n_{w}^{\infty}=V_{w} / V^{\infty}$ the final volume fraction of water. Here $V_{w}$ is the current volume of water, $V^{\infty}$ is the final volume of the soil mixture. The volume fraction $n_{w}^{\infty}$ can also be written

$$
n_{w}^{\infty}=\frac{w \rho_{g} / \rho_{w}}{1+w \rho_{g} / \rho_{w}}
$$

where $w$ is the water content of the soil, $\rho_{g}$ and $\rho_{w}$ are the mass densities of the solid particles and water respectively.

Figure 2 presents the stiffening curve relating the pressure (equal to the mean soil stress) to its density. The bulk modulus $K$ which corresponds to the slope of this curve progressively increases to finally be equal to the bulk modulus $K_{w g}(w)$ of a mixture of water and solid particles. From this point onwards, the bulk modulus of the crushed soil is constant. The expression includes two parameters, which are fixed by the bulk modulus at zero pressure and the knowledge of a particular point.

\section{Pressure and shock wave propagation in small scale buried charge experiments}

The detonation of a small quantity of explosive (100 g of C4) buried in a soil tank with either dry or saturated STANAG soil can now be addressed. The explosive 


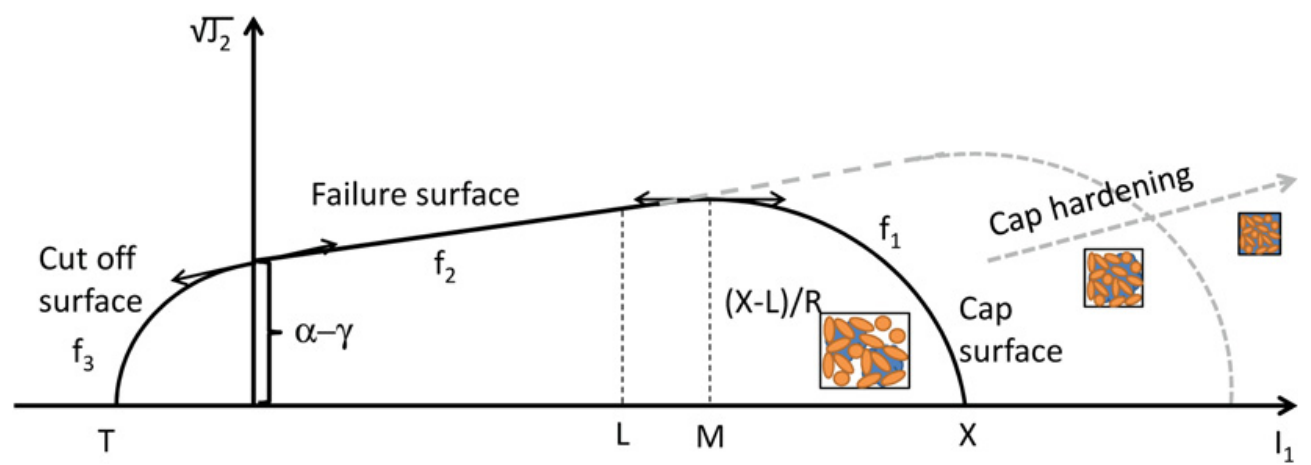

Figure 1. The yield surface of the soil model. The region of the cap is the only one which can harden.

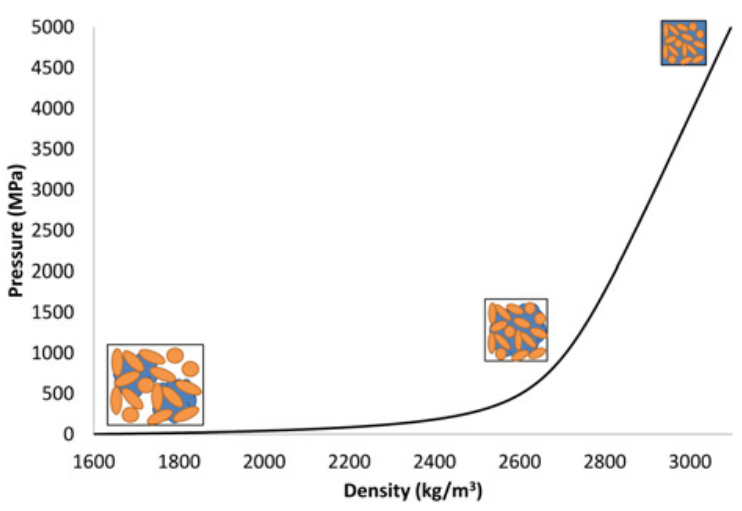

Figure 2. Curve relating the mean soil stress $p$ to its density $\rho$ underlining the increase of the bulk modulus $K$ of the soil.

was $20 \mathrm{~mm}$ high and had a diameter of $64 \mathrm{~mm}$. It was surrounded by polystyrene to maintain the detonator in place.

Three depths of burial were tested: a flush charge (its top surface is at the same level as the free surface of the soil), and charges buried at 3 and $8 \mathrm{~cm}$. The depth of burial was measured between the top surface of the explosive and the free surface of the soil.

Two states of the soil were considered: a STANAG soil with its natural water content (the soil was stored in the field) and a saturated STANAG soil.

A soil tank of $1 \mathrm{~m} \times 1 \mathrm{~m} \times 0.5 \mathrm{~m}$ was designed so as to mimimize the quantity of soil to be prepared while limitating the influences of boundaries. Particular attention was paid to the soil preparation. To obtain acceptable reproducibility, the same methodology was followed for all the shots. The soil was prepared in two layers. Each of them was moistened (for a saturated soil) and tamped. Tamping is used to increase the mass density.

The density and the water content were controlled in two ways. A sample was extracted from the soil tank. Its weight was measured to obtain its density and it was oven dried to get its water content. A gamma densitometer was also used. This nuclear device can measure both density and water content. A probe is placed in the soil and two different sources are emitted: Cesium 137 and Americium 241 are sent from the probe. The first one provides the density of the soil: gamma rays are scattered when colliding with soil particles. The dispersion of gamma rays is directly linked to the density. As for the water content,

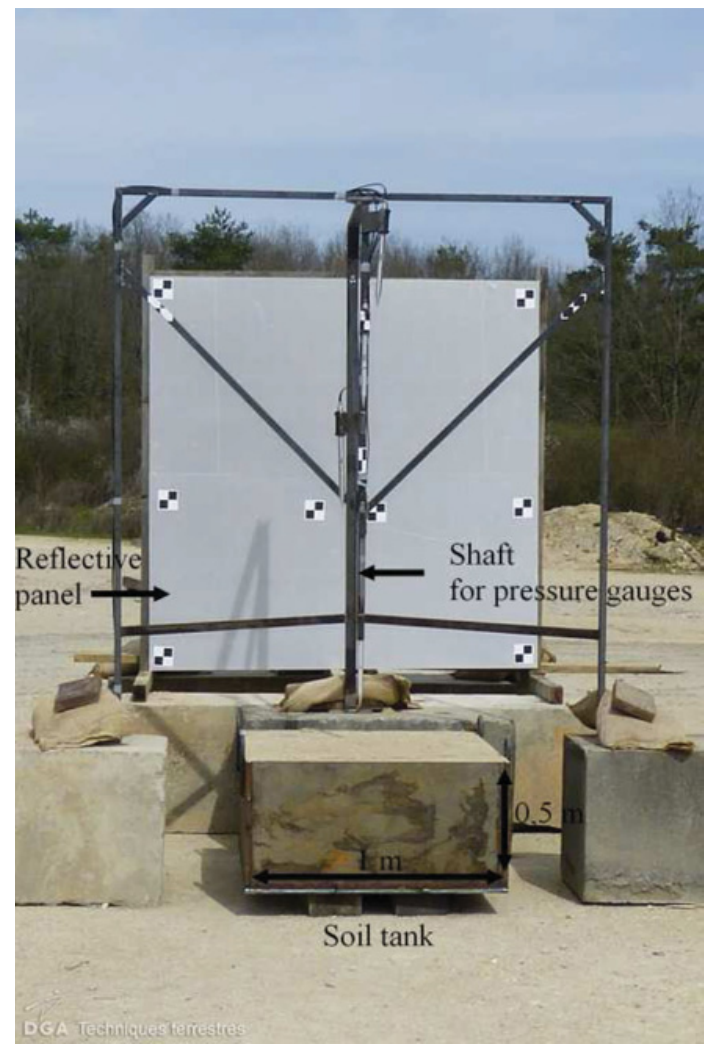

Figure 3. Experimental setting of small charge experiments.

the fast neutrons from Americium 241 are naturally slowed down by hydrogen atoms. Since water contains the largest quantity of hydrogen atoms in a soil, the number of slow neutrons can be related to the water content of the soil.

After each shot, the resulting crater was scanned to get its profile.

Figure 3 presents an overview of the experiments. A shaft was mounted above the soil tank to place five pressure gauges. They were located at 30, 70, 110, 150 and $190 \mathrm{~cm}$ above the free surface.

A reflective panel was placed in the background. The shock wave drives a difference of density. Its reflection on the reflective panel can be followed by a high speed camera. A rate of 20000 pictures per second was chosen. Figure 4 shows the resulting shock wave propagation observed for a detonation of a flush explosive in a dry sandy gravel, and for a detonation of an explosive buried 


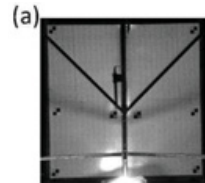

$\mathrm{T}=50 \mu \mathrm{s}$

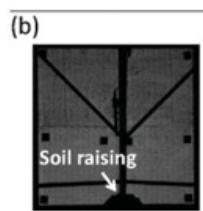

$\mathrm{T}=636 \mu \mathrm{s}$

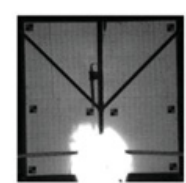

$\mathrm{T}=250 \mu \mathrm{s}$

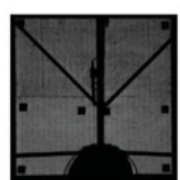

$\mathrm{T}=886 \mu \mathrm{s}$

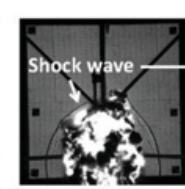

$\mathrm{T}=650 \mu \mathrm{s}$

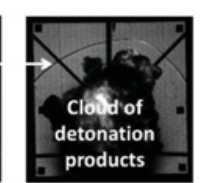

$\mathrm{T}=1550 \mu \mathrm{s}$

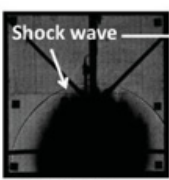

$\mathrm{T}=1886 \mu \mathrm{s}$

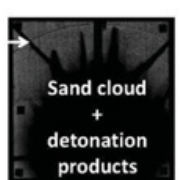

$\mathrm{T}=3386 \mu \mathrm{s}$
Figure 4. Pictures taken at 4 instants during the detonation (a) in dry sandy gravel for a flush explosive and (b) in a saturated soil for an explosive buried at $3 \mathrm{~cm}$.

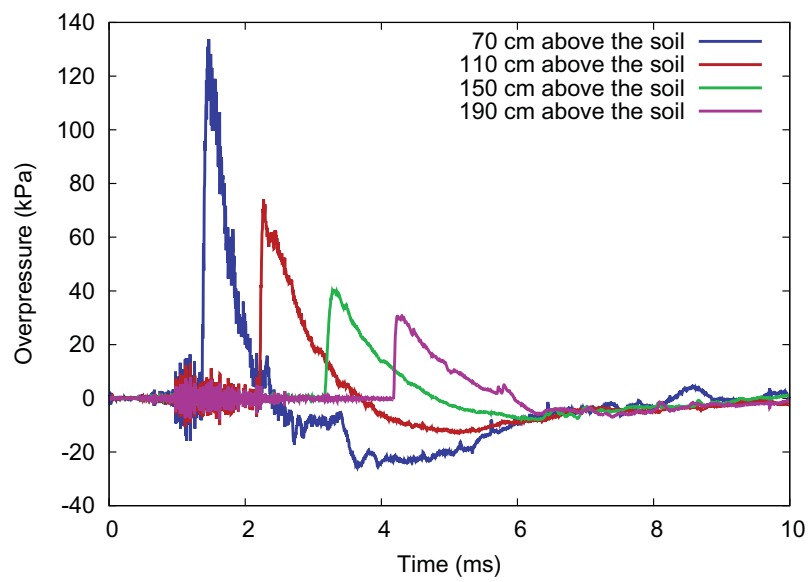

Figure 5. Pressures obtained at the four highest gauges above the soil surface for an explosive buried at $8 \mathrm{~cm}$.

at $3 \mathrm{~cm}$ in a saturated soil. For the detonation of a flush explosive, the light of the detonation is first visible, then the shock wave followed by the detonation products appears. In the case of a buried explosion, the light cannot be observed anymore since it occurs in the soil. At variance the raise of the soil is first clearly visible. Then the shock wave propagates ahead of a cloud composed of detonation products and soil projections.

Figure 5 presents the pressures measured at the four highest gauges of the shaft for the detonation of a charge buried at $8 \mathrm{~cm}$. Apart from a vibration of the structure which appears at the beginning of each signal, the rest of the measures is not disrupted by soil projections. On the other hand, the first pressure can be very noisy due to the impacts of soil particles on the gauge.

The spatial decrease of the maximum pressures is exponential. The maximum pressures plot almost linearly in a logarithm scale in Fig. 6. The pressures for a buried charge at $3 \mathrm{~cm}$ are more scattered than for a depth of $8 \mathrm{~cm}$. The STANAG soil contains grains with a size up to $4 \mathrm{~cm}$. For a depth of burial smaller than this maximum size, the aerial pressures may depend on the way the soil is replaced on the explosive and how the grains are arranged.

Except on crater sizes where differences could be noticed between dry and saturated soils, the pressures show negligible difference between unsaturated and saturated.

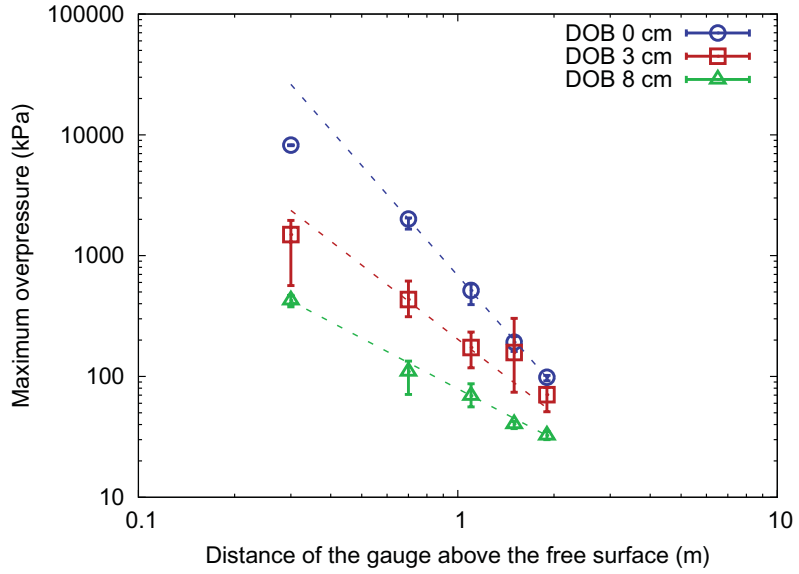

Figure 6. Maximum overpressures in logarithmic scale obtained at 5 heights for 3 depths of burial (DOB): a flush charge and charges buried at 3 and $8 \mathrm{~cm}$.

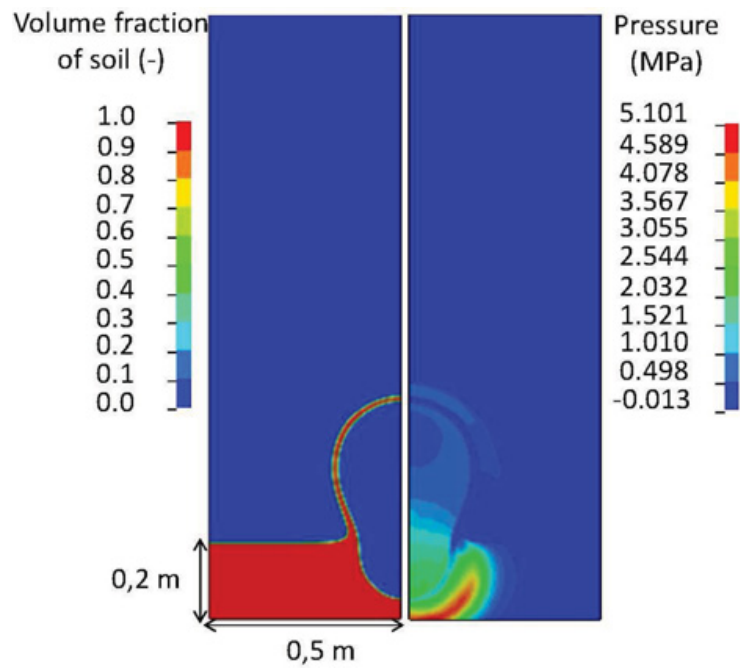

Figure 7. Contours of volume fraction of soil (left) and pressure (right) at $0.5 \mathrm{~ms}$ after the detonation of $100 \mathrm{~g}$ of $\mathrm{C} 4$ buried at $3 \mathrm{~cm}$. In the left plot, the red (resp. blue) color denotes the soil (resp. air).

\section{Small scale experiments versus simulations}

Axisymmetric Eulerian simulations using the viscoplastic cap model have been run with LS Dyna to reproduce the experiments. The simulation outputs were the pressures at the five heights above the soil. While the explosive was modelled with a JWL equation, a polynomial equation was chosen for the air part. The polystyrene surrounding the explosive was taken into account with the same dimensions as in the experiments considering a space of air around the explosive. The explosive was initiated at its bottom center. As for the boundary conditions, a condition of zero displacement was chosen at the border of the soil to take into account the reflections on the soil tank while the atmospheric pressure was applied on the free surface.

Figures 7 and 8 show the contours of pressure and of volume fraction of soil at $0.5 \mathrm{~ms}$ and $1.5 \mathrm{~ms}$ after the detonation of $100 \mathrm{~g}$ of $\mathrm{C} 4$ buried at $3 \mathrm{~cm}$. The 


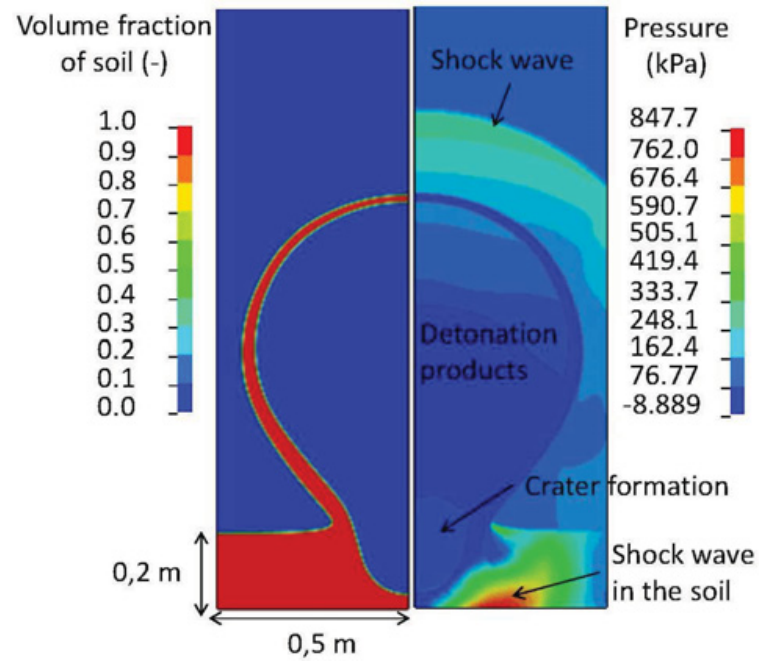

Figure 8. Same as Fig. 7 but at $1.5 \mathrm{~ms}$ after the detonation.

simulations reproduce qualitatively and quantitatively well the phenomena that are obversed during the experiments: since the explosive is buried, a soil cap moves upward and is visible in pressure contours (darker blue) and in the volume fraction creating a kind of soil bubble containing the detonation products. As time goes by, the bubble grows up until it has reached a maximum height and it subsequently drops. Since no fracture model has been introduced, the bubble remains closed during the whole simulation, and the detonation products cannot escape.

As in experiments, once the shock wave has reached the free surface, it propagates in the air in front of the soil projections. In the simulations, the shock wave is clearly noticeable on the pressure contours, while the motion of the soil, in particularly above the explosive can be deduced by the volume fraction of soil.

As indicated by the pressure contours, the soil undergoes large stresses. The elastic stiffening of the soil makes it able to resist the detonation. If the elastic moduli had kept a standard and fixed value throughout the simulation, the soil would have not resisted and would have been pushed away by the detonation.

The mesh size was determined with a sensitivity study. The simulation of the detonation of a flush explosive requires a finer mesh than for a buried charge. In the soil, the energy dissipation is more important than in the air, resulting in a faster widening of the shock wave. Since several elements must be contained in the shock wave, a coarser mesh could be selected to model buried detonations. Therefore the mesh sizes were equal to $1 \mathrm{~mm}$ and $5 \mathrm{~mm}$ for flush charges and buried charges respectively.

The influence of each parameter of the model on the pressures was studied. Two parameters were found to be very important, namely the initial density and the size $X$ of the yield surface.

The relative size of the explosive is small compared to the thickness of the soil placed back above the charge. This is particularly the case when the $20 \mathrm{~mm}$ thick charge is buried at $8 \mathrm{~cm}$. The initial density of the soil plays an important role: a denser soil requires a larger part

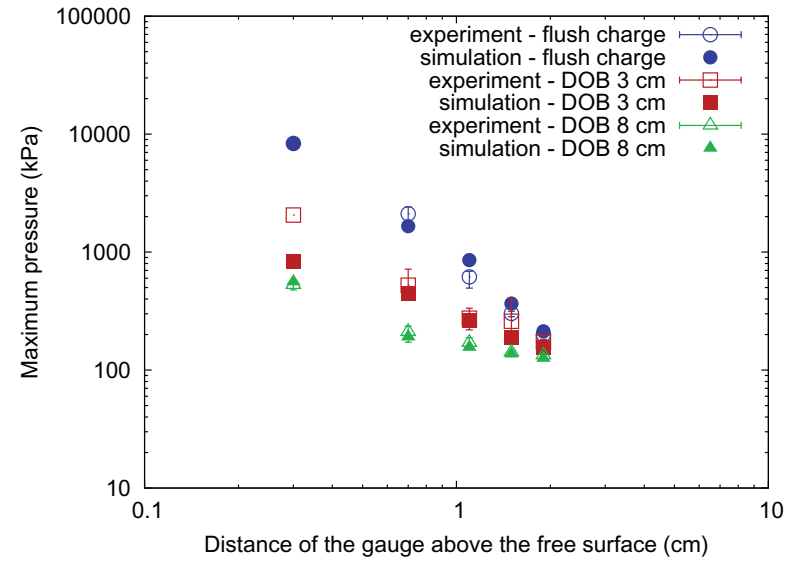

Figure 9. Maximum pressures for simulations and experiments at five heights above the soil surface for three depths of burial $(0,3 \mathrm{~cm}$ and $8 \mathrm{~cm})$.

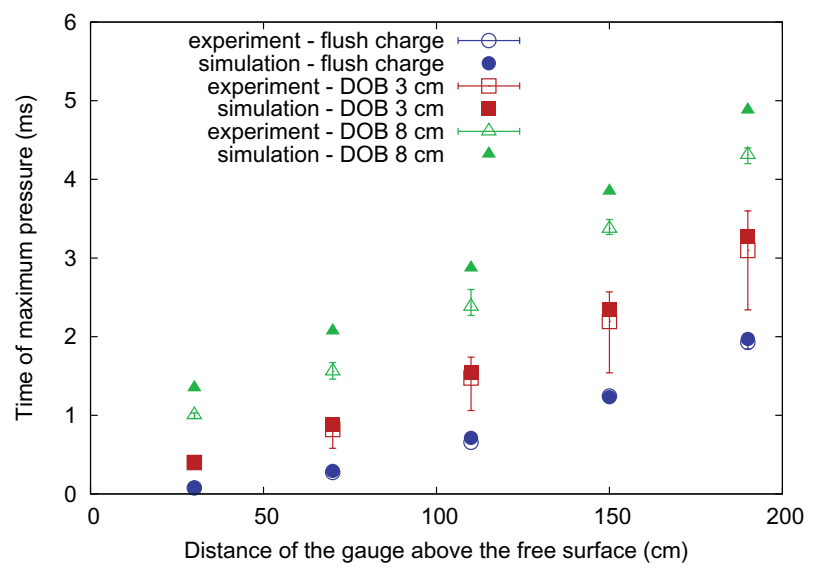

Figure 10. Times of arrival of maximum pressures between simulations and experiments at five heights above the soil surface for three depths of burial $(0,3 \mathrm{~cm}$ and $8 \mathrm{~cm})$.

of the explosive energy to be spent in its deformation. Correspondingly, the resulting aerial pressures are smaller than those emanating from a lighter soil.

As the size $X$ of the yield surface increases, the soil is more elastic. The speed of propagation of the shock wave is increased. Larger pressures result and the arrival of the shock wave is faster.

The maximum pressures and their corresponding times of arrival are compared to the small scale experiments for the three depths of burial in Figs. 9 and 10.

For a flush charge and a charge buried at $3 \mathrm{~cm}$, the simulations reproduce well the experiments. For a charge buried at $8 \mathrm{~cm}$, while the pressure outputs are similar to the experiments, the simulation outputs are slightly delayed with respect to the experiments. Since it remains constant for the five gauges, this delay may be due to soil preparation.

\section{Experimental study of impulse and plate deformation}

The above small scale experiments provide essentially aerial pressures. A second experimental campaign aimed 
at evaluating the impulse transmitted by larger masses of explosive to a test rig and at measuring the deformation of plates which stand for vehicle bellies.

Various parameters linked to the soil and to the plate were tested: two depths of burial (100 and $250 \mathrm{~mm}$ ), two stand off distances measured between the plate and the soil (600 and $800 \mathrm{~mm}$ ), various thicknesses of armored steel plates $(15,20,25,30$ and $50 \mathrm{~mm})$ and various masses of explosives (2, 4, 6 and $8 \mathrm{~kg}$ ) were studied.

In the first experimental campaign the aerial pressures emanating from a soil stored in the field and a saturated soil did not show much differences. At variance in this campaign, the water contents were clearly distinct, which had noticeable consequences on deformations and on impulses.

\section{Conclusion}

Small scale experiments have been carried out to provide data for simulations of the explosions of buried landmines. Pressures were measured at various heights above the free surface of the soil where the explosive was buried at three different depths in a dry or saturated soil. However, the dry soil was stored in the field. So the difference on water content between what was called a dry soil, and a saturated soil is not large enough to influence the pressures significantly.

A model of soil has been developed. It takes into account the stiffening of the soil due to the shock wave and the water content. The outputs of pressure in the simulations were compared to the measurements. They correlate well the experiments. For the charge buried at $8 \mathrm{~cm}$, the time of arrival of the shock wave predicted by the simulations is delayed with respect to the experiments.
This delay may be due to the way the soil is placed on the top of the explosive.

A second experimental campaign with larger explosive masses has been launched with emphasis on impulse and plate deformation. A dedicated test rig has been developed. Further finite element analyses will be run to simulate the measurements obtained during these large scale experiments.

\section{References}

[1] Hlady S. L., 18th Military Aspects of Blast and Shock (2004)

[2] Peles S., Touati D., Azulay I., Neuberger A., 24th International Symposium on Ballistics (2008)

[3] Fourney W. L., Leiste H. U., Hauch A., Jung D., IMPLAST Conference (2010)

[4] Zakrisson B., Haggblad H. A., Jonsen P., Central European Journal of Engineering 2, 532-550 (2012)

[5] Assaf Z., Ran E., Golan G., Drori O., Katalan I. K., 28th International Symposium on Ballistics (2014)

[6] Bergeron D., Walker R., Coffey C., Detonation of 100-Gram Anti-Personnel Mine Surrogate Charges in Sand (DRES, Canada, 1998)

[7] AEP-55, Procedures for evaluating the protection level of logistic and light armoured vehicles, Volume 2, Edition C, Version 1 (NATO, 2014)

[8] Tong X., Tuan C., Journal of Geotechnical and Geoenvironmental Engineering 133, 206-214 (2007)

[9] Livermore Software Technology Corporation (LSTC), LS-DYNA theoretical manual (Livermore, California, 2014)

[10] De A., Computers and Geotechnics 43, 72-79 (2012) 